\title{
Position change in cognitive conflict as a function of the cue-criterion relationship and the initial conflict
}

\author{
DANIEL DRUCKMAN, Institute for \\ Juvenile Research, Chicago, Ill. 60611
}

A complex simulation was designed to explore the effects of variations in two types of differences between parties on position change in a cognitive conflict. The nature of the cue discrepancy and the distance between initial predictions of a criterion variable were found to affect measures of position change and willingness to compromise. $A$ discrepancy in cue weightings that served to polarize parties ideologically resulted in resistance to yielding; also, parties were more willing to compromise on the issue characterized by the shortest distance between initial positions.

Students of interest conflicts have been concerned with the design of techniques for increasing the "fund" of shared interests between opponents as a means for reducing the intensity of conflict between them over preferred outcomes in resource distribution. Such consolidating techniques as superordinate goals (Sherif et al, 1961) and crosscutting overlapping loyalties (Coser, 1956) have been suggested as means for reducing polarization between contestants. However, the successful utilization of techniques for building cohesive communities of shared interests does not preclude division along other lines. Attempts at increasing intergroup or interpersonal cooperation may have the effect of producing conflict over the best means for accomplishing the shared goal. While differences over preferred means for solving a problem may be linked to a conflict of interest, the two types of conflict have been distinguished conceptually. Conflicts of interest result primarily from different outcome preferences rather than from beliefs in the efficacy of alternative methods for reaching the same end. The latter type of cognitive conflict is most likely to occur when there are several plausible alternative procedures available for achieving a goal and when criteria for evaluating achievement are ambiguous. This study employed such a situation, in the setting of political decision making, in order to investigate the effects of certain variables on attempts to resolve a cognitive conflict.

Hammond (1965) designed a laboratory model of cognitive conflict to complement previous investigations of conflict, which used a conflict-of-interest paradigm. Based on Brunswik's probabilistic functionalism, the cognitive conflict model presumes a rational organism who bases his decisions on the results of processed data. Individual preferences for predictions of a level of a criterion variable, such as "the amount of democracy in a number of countries" or "the amount of school integration in a number of cities," are learned on the basis of observed relationships between presumed causes of an event (cues) and values of the criterion variable. The mode specifies differences between contestants at two levels: in cue-criterion relationships established through laboratory training or prior experience and in the actual prediction of the criterion variable. Position difference is defined by the distance between the two contestants' predictions of a criterion. (In conflicts of interest, the discrepancy between initial positions is conceived of in terms of the extent to which the order of preferences for the range of possible outcomes is reversed.) The initial conflict between predictions derives directly from experimentally programmed differences in the cue-criterion relationships learned in the laboratory. During training, Ss are given an opportunity to learn the cue-criterion relationships by examining different samples of materials. Different relationships are learned, depending on the particular sample of materials seen by each of the Ss. In the conflict resolution task, the parties have the common goal of achieving an agreed-upon solution to the problem of predicting a criterion value from new data. Cognitive conflict is not explored in a mixed-motive situation; participants are rewarded as a dyad for making an improved prediction of the criterion. In earlier applications of the paradigm, by Hammond and his colleagues, the conflict between Ss over cue weightings (or differential preferences for means) do not derive from different ideological orientations, nor are they linked to a conflict of interest.

Among the factors hypothesized to influence attempts by Ss (whose training is not identical) to reconcile the differences in their initial predictions of a criterion value are the nature of the discrepancy in the cue-criterion relationship and the amount of initial conflict that results from their different training. Hammond et al (1966) reported that the nature of the relationship between cues and the criterion (viz, linear vs nonlinear) interacts with the amount of initial conflict in effecting measures of compromise and cognitive change. For example, Ss trained on cues that related to a criterion in a nonlinear fashion compromised less in private when the initial conflict was large. [Summers (1968)found that Ss who based their predictions of the status of a nation's minority citizens a generation into the future on only one cue (viz, educational opportunity), compromised more than did Ss who based their predictions on two cues (viz, educational and legislated opportunity).] With regard to initial conflict, Summers (1968) found that compromise tended to be inversely proportional to the initial difference between the Ss' judgments, although the relationship was weak. However, in these studies, the amount of initial conflict was not controlled or systematically varied by the investigator. Initial position differences in the prediction of a criterion value were generated directly from the nature of the cue-criterion relationship learned by the Ss. The effects of position differences were not assessed independently of the effects of the nature of the cue-criterion relationship. A purpose of this study is to determine the relative effects of the nature of the cue-criterion relationship and the size of initial position differences on measures of willingness to compromise and position change.

The amount of cue discrepancy between Ss was investigated by Rappoport (1965). He found no difference between high and low cue-discrepancy conditions on measures of the tendency to compromise. In this study, amount of cue discrepancy was varied in a task involving conflict over means for solving social problems. The interpersonal problem-solving tasks used in previous experiments required $\mathrm{Ss}$ to make predictions of several criterion values, represented as trials, but permitted only a minimum of debate over the opposing predictions. In this experiment, a cognitive conflict was embedded within a complex simulation of political decision making. Participants, playing roles of political decision makers, were permitted to debate their opposing predictions on several criterion variables for a maximum of $30 \mathrm{~min}$.

The conflict was created between two simulated city councils in the same metropolitan area. Participants, playing roles of representative decision makers from opposing factions (councils). were to attempt to reconcile their different predictions of the amount of urban unrest to be expected during the following summer in four communities. The 
simulated city councils were competing with other cities for a model-cities grant to be awarded by the federal government to the metropolitan area whose representatives made the most accurate predictions of urban unrest in each of four communities. Thus, it was necessary to agree on a prediction for each of the communities but not to forfeit accuracy in the process.

Each simulation session consisted of two three-person councils; assignment of participants to teams was randomly determined. The simulation consisted of three phases, each representing a "compressed-time" analogue of a process hypothesized to characterize decision making among political representatives with opposing viewpoints. In the first phase the two teams were separated in order to process, independently, census-type data for determining the major causes of urban unrest. Data were presented for each of 10 variables (cues) hypothesized in the "scenario" to relate to urban unrest in $\mathbf{1 0}$ hypothetical communities. (Also, information on population, area, average income, and growth rate were included.) The raw data consisted of scaled values for each variable and a value for a criterion variable, number of reports of urban violence. A description of each variable, including the criterion index, and the nature of the scale constructed for rating each community was provided. From the values provided for each community, the "decision makers" were to derive a rank order of the variables in terms of their relation to the "index of urban unrest." Mean difference scores between cue values and criterion values, obtained across the 10 communities, were ranked. The first four variables, in terms of rank, were then jsolated and used for making predictions of values on the "index of urban unrest" for four new communities. As a result of receiving data from a different sample of communities, the two city council factions came up with a different rank ordering of the same variables or with different variables.

In the second phase, the two councils were to use the four isolated causes for deriving predictions of the amount of unrest to be expected the following summer in each of four communities. Following this, time was allotted for brief within-team caucusing in order to prepare a "position paper" that would provide rationale for the four isolated causes to be defended in the debate session. The procedure consisted of assigning weights, ranging from 4 to 1 , to each of the cue values in terms of its rank and multiplying these values by the weight. The weighted values were then added, and the sum was converted to a value on the 10-step criterion scale, ranging from 20 to 500 reports of urban unrest. These values represented the prediction made by each council for each of the hypothetical communities ("Bering Heights," "Astor," "Melville," and "New Cardonia"). The cognitive conflict was defined by the discrepancy between the predictions made by the opposing factions.

The third phase of the simulation consisted of a 30-min debate session between representatives of the two factions in order to resolve their opposing predictions. The session ended earlier if decisions were reached for all four communities before the 30 -min deadline. All members from both teams engaged in the decision-making discussions; a member from each team was paired randomly with an opposite number and the three dyads were separated during the debate.

The manipulated variables consisted of the nature of the discrepancy between opponents in cue weightings and the distance between the initial predictions of the criterion variable, "number of reports of urban unrest." Three types of cue discrepancy, considered as an independent variable, were made orthogonal to four levels of position differences, considered as a repeated measure. The largest programmed discrepancy consisted of a partial nonoverlap of cues. The opposing teams shared two out of four predictor variables. The other two programmed discrepancy conditions made use of the same four variables: density index, relative socioeconomic status index, community organization index, and an index of police brutality. In one condition, the ranks were reversed, i.e., density and relative socioeconomic status were ranked first and second for one faction and fourth and third, respectively, for the other. In the other condition, the cues were only partially reversed, i.e., density was first for one faction and second for the other, police brutality was third for one and fourth for the other, etc. This condition is referred to as the minimum discrepancy condition. The distance between the predictions made by each team varied for each community but was the same across the three cue-discrepancy conditions. These were $8,12,16$, and 20 steps on a 20-step scale of "number of reports of urban unrest." For each session, the order of presentation of the scales was determined randomly.

Each session was homogeneous by sex; an attempt was made to balance the male-female ratio across the conditions. Across all of the conditions, 44 dyads (Northwestern University undergraduates assigned randomly to a condition and to a team) were used in the analysis. The

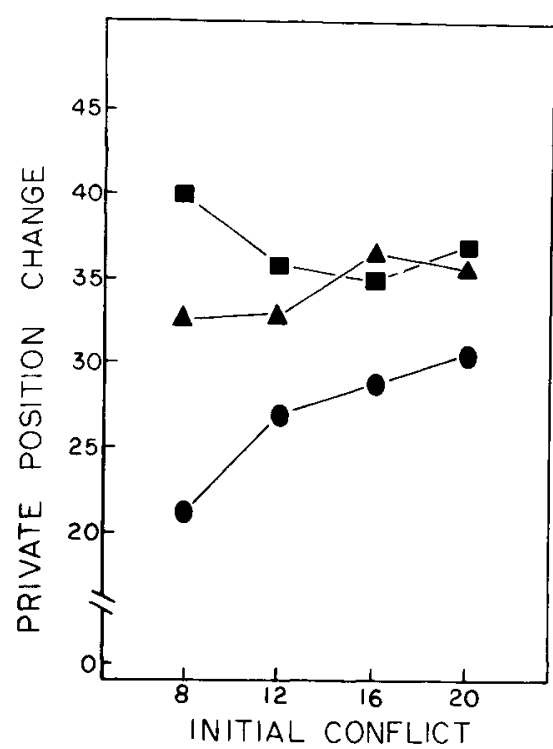

Fig. 1. Private position change as a function of amount of initial conflict and type of cue discrepancy: minimum

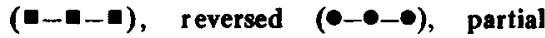
nonoverlap ( $\wedge \subset \wedge<)$.

was as follows: 18 in the reversed condition, 12 in the minimum condition, and 14 in the partial nonoverlap condition. Teams that did not complete the computations correctly or comply with the instructions were excluded from the analysis.

\section{RESULTS}

The effects of the manipulations were assessed on three dyadic measures: private position change, amount of consensus, and willingness to compromise. At the conclusion of the debate, Ss were asked to indicate, privately, their best prediction of the amount of unrest in each of the four communities. They were also asked to indicate, as a result of the debate, the maximum amount of movement that they were willing to yield from their initial positions in order to get an agreement with their opponent. The measure of private position change consisted of the ratio of the amount of movement from an initial position to the amount of initial distance between positions. The mean of the ratios for two members of the same dyad was used as a dyadic measure in a two-way 4 by 3 analysis of variance with one repeated measure (see Winer, 1962, pp. 306-310). The results indicate a significant cue-discrepancy main effect $(\mathrm{F}=4.11, \mathrm{df}=2 / 132, \mathrm{p}<.05)$. The initial conflict effect and the interaction did not approach significance. Application of the Newman-Keuls method for individual comparison of means (see Winer, 1962, p. 309) indicates that the "reversed" 
cue-discrepancy condition differs significantly from the other two conditions. This difference is illustrated in Fig. 1. Across all levels of initial conflict, participants in the "reversed" cue-discrepancy condition yielded less from their initial position than those in the "minimum-discrepancy" and "partial-nonoverlap" conditions. Amount of consensus was defined as the ratio of the scale distance between the two opponents' private judgments of the best prediction to the amount of initial distance between positions. The results of a two-way analysis of variance with one repeated measure indicates a significant main effect for initial conflict $(\mathrm{F}=3.26, \mathrm{df}=3 / 123, \mathrm{p}<.05)$. While the differences between the means were in the same direction as on the measure of private position change, the cue-discrepancy effect did not attain significance $(F=2.05, \quad d f=2 / 132$, $\mathrm{p}>.05)$. The interaction was not significant. The Newman-Keuls method for individual comparisons indicates that the smallest initial conflict (eight steps) resulted in significantly more consensus than the other three conditions $(12,16$, and 20 steps). Similar results were obtained for the measure of willingness to compromise. This index was defined as the ratio of the difference between how far each participant was willing to go to get an agreement and his best prediction to the amount of initial distance between positions. The mean of this index for the two members of the same dyad was used in the analysis of variance. The initial conflict main effect was highly significant $(F=5.90, \mathrm{df}=3 / 123, \mathrm{p}<.01)$, indicating that participants were most willing to yield from their best prediction to get an agreement on the issue characterized by the smallest initial conflict. Other differences were not significant.

\section{DISCUSSION}

The two types of differences between opposing parties to a cognitive conflict, specified by the "Hammond paradigm," are the nature of the discrepancy in the cue-criterion relationship and the distance between the initial predictions of a criterion variable. The evidence presented in this paper indicates that variations of these types of differences affect measures of position change and willingness to compromise in order to reach agreement on a prediction. To ensure that yielding was not a function of scale distance per se, measures were corrected statistically for initial scale size. While the relationship between initial conflict and yielding was not linear, the most movement from initial positions on measures of willingness to compromise and consensus on a "best" prediction occurred on the issue for which the initial distance between positions was smallest. This finding perhaps suggests that the initial distance between predictions is an index of the intensity of the conflict.

The "reversed" cue-discrepancy condition resulted in the least private yielding from initial positions across the four levels of initial position differences. The finding of no difference between the "minimum" and the "partial nonoverlap" conditions indicates that the results are not due to the amount of discrepancy between the cue weightings. However, another dimension of difference between the conditions is the nature of the division between opponents. The importance of this dimension is suggested by additional data collected in connection with this study. Among the postnegotiation reactions elicited from the participants following the debate was a question on the perceived incompatibility of the starting positions. Dyads in the "reversed" cue-discrepancy condition saw their initial positions as significantly more incompatible $(\bar{X}=2.00)$ than those in the "minimum" discrepancy condition $(\bar{X}=2.40)$ and those in the "partial nonoverlap" condition $(\overline{\mathrm{X}}=2.45)$. The observed differences in "perceived incompatibility" may reflect a difference in debate process between the conditions. In postnegotiation reactions, participants in this condition noted that the discussion focused on an ideological division concerning preferred causes of social problems. One party emphasized cues that were considered as underlying causes of urban tension which would demand long-term programs for their solution (i.e., density and relative socioeconomic status) while the opposing party, weighing the same cues in reverse order, emphasized contemporaneous causes which would demand relatively short-term plans to deal with (i.e., community organization and police brutality). An emphasis on underlying causes implies solution attempts that involve changes in the social structure, while an emphasis on immediate causes implies solutions that would not effect the institutional structure of society. This polarization, created by the programmed reversed cue-preferences, perhaps accounted for the observed resistance to yield from initial positions characteristic of this condition. The programmed differences created in the other two cue-discrepancy conditions did not result in a polarization along ideological lines, i.e., both parties emphasized both an "underlying" and an "immediate" cause.

The cognitive conflict paradigm was designed to study differences between parties in empirically-derived predictions concerning the most workable solutions to a problem. However, cognitive conflicts may also result from differences in ideological beliefs concerning the most desirable solutions. The salience of ideological divisions in conflicts over means for solving social problems adds a dimension to the cognitive conflict model. An implication of these results is that ideological divisions may be an important source of resistance to yield from initial positions (see Coser, 1956, p. 118). However, additional experimentation is necessary to clarify this issue.

\section{REFERENCES}

COSER, L. A. The functions of social conflict. Glencoe, Ill: Free Press, 1956.

HAMMOND, K. R. New directions in research on conflict resolution. Journal of Social Issues, $1965,11,44-66$.

HAMMOND, K. R., TODD, F. J., WILKINS, M., \& MITCHELL, T. O. Cognitive conflict between persons: Application of the "Lens Model" paradigm. Journal of Experimental Social Psychology, 1966, 2, 343-360.

RAPPOPORT, L. H. Interpersonal conflict in cooperative and undertain situations. Journal of Experimental Social Psychology, 1965, 1, 323-333.

SHERIF, M., HARVEY, O. J., WHITE, B. J., HOOD, W. R., \& SHERIF, C. W. Intergroup conflict and cooperation: The robbers cove experiment. Norman, Okla: University Book Exchange, 1961.

SUMMERS, D. A. Conflict, compromise, and belief change in a decision-making task. Journal of Conflict Resolution, 1968, 12, 215-221.

WINER, B. J. Statistical principles in experimental design. New York: McGraw-Hill, 1962. 